\title{
Applied Fracture Mechanics in Metallurgical Failure Investigations
}

\author{
M.E. Stevenson and J.L. McDougall \\ Metals \& Materials Engineers, Suwanee, GA, USA 30024
}

The practice of metallurgical failure analysis frequently necessitates the detailed investigation of metal fractures. During the course of such investigations, it is common practice to identify the mechanism and pathway by which fracture occurred. While identification of the fracture mechanism is a vital aspect of fracture related analyses, it is often not sufficient to fully determine the root cause(s) of a particular failure. In many instances, a greater depth of information beyond identification of the fracture mechanism is required, and evaluation of the macro scale mechanical and environmental conditions may be necessary.

While fractographic and metallographic observations can provide insight into the state of stress and operating environment and the mechanism by which failure occurred, specific linkages to the operating variables that influenced failure may not be addressed. These examinations are staples of metallurgical failure investigations and are frequently provide the primary data from which root cause determinations are made. By coupling fractographic and metallographic observations with quantitative techniques such as fracture mechanics, a greater understanding of the failure can be gained.

This paper outlines the fundamentals of applying fracture mechanics during the course of metallurgical failure investigations. The linkage between data gathered from fractographic and metallographic investigations and their usage in fracture mechanics studies will be addressed. Data collection and interpretation, specifically with respect to spatial orientation relative to the fracture location will be discussed. Additionally, selection of metallographic specimens and development of sectioning plans to maximize the utility of data for fracture mechanics analysis is addressed. The fundamental concepts are illustrated through several case studies that highlight the value of applying fracture mechanics as an aid to determine root cause. 


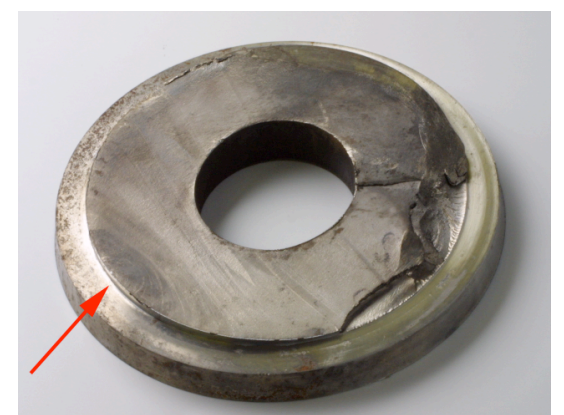

Figure 1. Fatigue fracture surface of a high speed shaft.

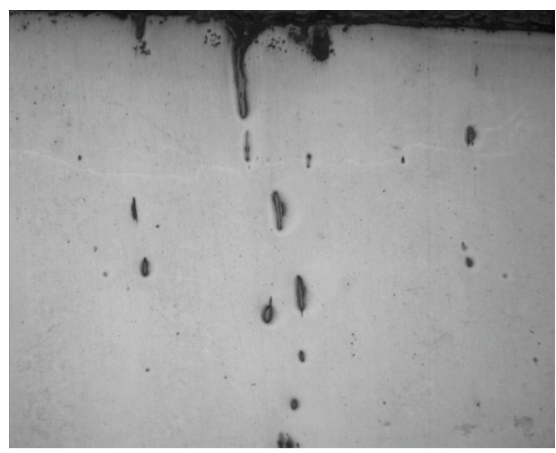

Figure 2. As polished micrograph (100x original magnification) of shaft just below the fracture plane.

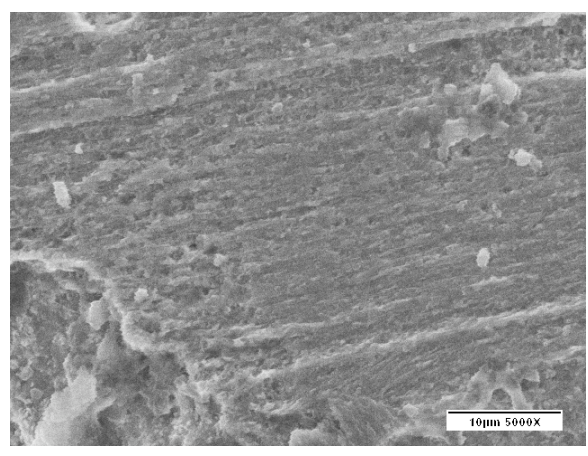

Figure 3. SEM micrograph of fatigue striations. Striation spacing measurements were utilized in determining the operating stress prior to failure, during the crack growth process.

References

[1] Hertzberg, Richard W. Deformation and Fracture Mechanics of Engineering Materials. $4^{\text {th }}$ ed. New York, NY: J. Wiley \& Sons, 1995.

[2] Bates, R.C., Clark, W.G. "Fractography and Fracture Mechanics." Transactions of American Society for Metals 62 (June 1969): 380-389.

[4] Irwin, George R., Paris, Paul C., Tada, Hiroshi. The Stress Analysis of Cracks Handbook. $3^{\text {rd }}$ ed. New York, NY: The American Society of Mechanical Engineers, 2000. 